\title{
Performance and Structural Load Analysis of Small and Medium Wind Turbines Operating with Active Speed Stall Control versus Pitch Control
}

\author{
Arash E. Samani ${ }^{* 1,2}$, Nezmin Kayedpour ${ }^{1,2}$, Jeroen D. M. De Kooning ${ }^{1,3}$, Lieven Vandevelde ${ }^{1,2}$ \\ ${ }^{1}$ Department of Electrical Energy, Metals, Mechanical Constructions and Systems, Ghent University, Belgium \\ ${ }^{2}$ EEDT Decision \& Control, Flanders Make \\ ${ }^{3}$ EEDT Motion Products, Flanders Make \\ Email: *EbnealiSamani.Arash@ugent.be
}

\begin{abstract}
The objective of this paper is to investigate the performance of pitch and active speed stall control, and their impact on the structural loads of small and medium wind turbines (SMWTs). Large wind turbines use blade pitching to limit the power at high wind speeds. For SMWTs, the cost and complexity of a blade pitching system are not justified, so that passive yaw, stall control, or a furling tail mechanism is used instead. However, the choice of a proper control concept is not straightforward for SMWTs. In this regard, it is important to take into account that the control strategy has a significant impact on the structural loads. Reducing the structural loads results in a longer lifespan and ensures the safe operation of the wind turbine in stormy wind gusts or fierce winds. In this study, a $10 \mathrm{~kW}$ wind turbine is operated with different control strategies to investigate the corresponding loads, both in uniform and turbulent winds. The simulation results show that controlling power around the rated value is feasible and stable for both control strategies. However, active speed stall control increases the bending moments at the blade root, while it lightens the tower base moments, in contrast to the pitch control.
\end{abstract}

Keywords-active speed stall control, pitch control, load analysis, small and medium wind turbines

\section{INTRODUCTION}

Wind turbines are one of the most promising sources of clean and renewable energy. Large-scale wind turbines are becoming more and more mature. However, their large size can be an obstacle on land, making them most suitable for offshore installation. Therefore, small and medium wind turbines (SMWTs) can be used where land space is more scarce. Also, they can be installed close to energy consumers. However, they are technologically less mature than large turbines. Nevertheless, the amount of SMWTs is rapidly increasing, and the market continues to mature. According to [1], the global installed capacity of SMWTs is expected to grow from 176.4 MW in 2017 to $446.0 \mathrm{MW}$ in 2026.

Since SMWTs are mostly installed on farms and near individual houses or small and medium companies, developing a system to enhance the energy production and guarantee their safe operation is necessary, especially in turbulent wind areas. SMWTs are mainly controlled by passive control strategies which have a low performance at high wind speeds. Passive stall control and furling mechanisms cause overshoots and lower power output in high wind speeds [2]. Therefore, passive control concepts are not always suitable for regions with strong and turbulent winds. Also, a pitching mechanism is not commonly included in small turbines due to the high cost. Therefore, torque control is often used to limit the output power in high wind speeds [3]-[11]. However, the consequence of using torque control on the structural loading is not sufficiently studied.

As indicated, different control strategies can be implemented to limit the power and the rotational speed in high wind speeds, but not all of these strategies mitigate the structural loads. In other words, limiting the power at high wind speeds does not necessarily reduce the loads on the turbine structure. The impact of the control system on the structural loads should be investigated and taken into account in the design process. In this paper, the performance of both pitch control and active speed stall control is examined in turbulent wind conditions. Their impact on the structural loads on the tower and blades is analysed to select the most advantageous control strategy for this class of wind turbines.

This paper is organized as follows. Section II describes the wind turbine generator system. Section III explains the control strategies for the whole operating region of the wind turbine. The performance of the control systems is compared in Section IV. The comparison between structural loads of different control concepts is discussed in Section V.

\section{WIND TURBINE GENERATOR SYSTEM}

Fig. 1 gives an overview of the studied wind turbine system. The wind turbine rotor (a) is directly coupled to the Permanent Magnet Synchronous Generator (PMSG) (b). The direct-drive PMSG allows variable speed operation to maximise the power coefficient $C_{p}$ over a wide range of wind speeds. The PMSG generates an AC voltage with a variable frequency and amplitude, which is converted to a DC voltage by a rectifier (c). The inverter (d) then injects a sinusoidal current into the grid (e).

A three-bladed wind turbine with a rated power of $10 \mathrm{~kW}$ and a rotor diameter of $5.8 \mathrm{~m}$ is selected from the available models in the FAST software package [12]. Fig. 2 shows the power coefficient of the wind turbine as a function of the pitch angle $\theta$ and the tip speed ratio $\lambda$. The maximum power 


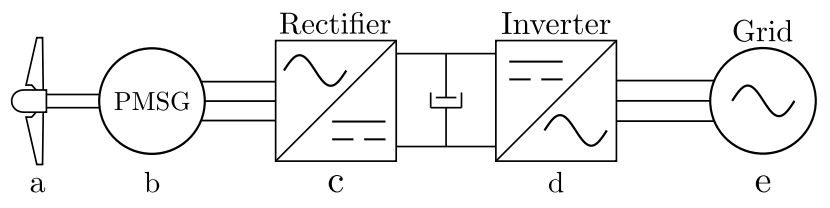

Fig. 1. Scheme of the direct-drive PMSG wind turbine system.

coefficient $C_{p}^{\max }$ is 0.42 at a tip speed ratio of 5.5 and a pitch angle of $7^{\circ}$. The nominal drivetrain efficiency is considered to be $90 \%$, which brings the rated electrical output power to $9 \mathrm{~kW}$.
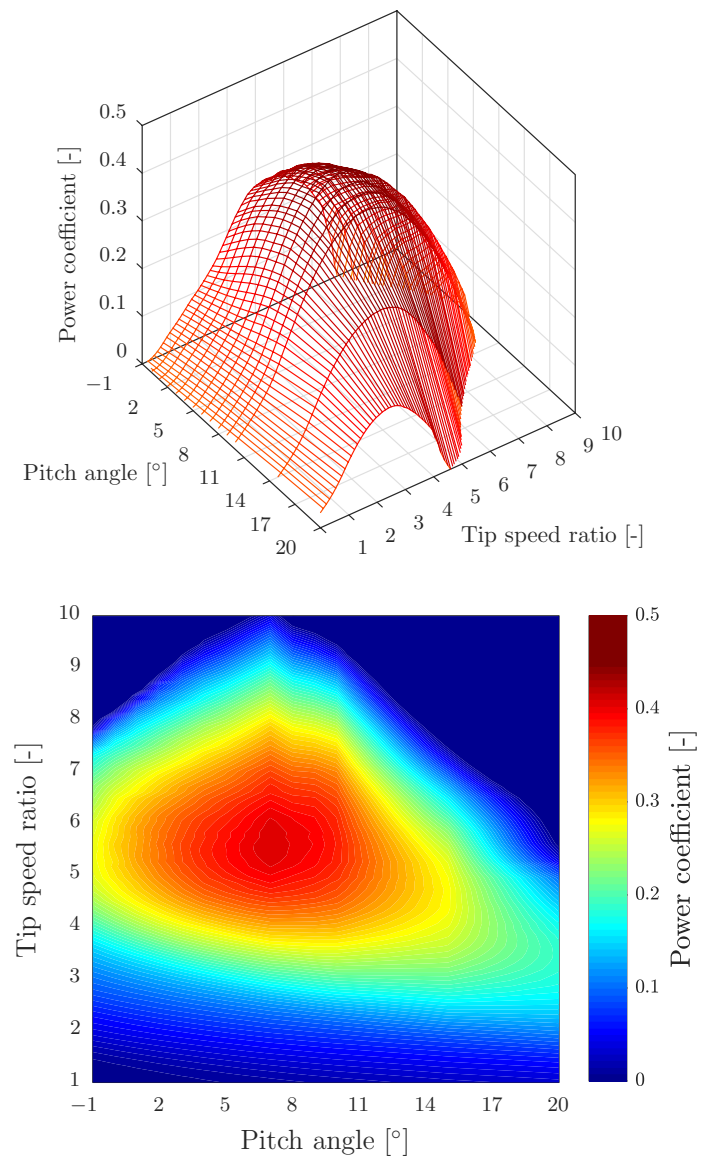

Fig. 2. Power coefficient as a function of pitch angle and tip speed ratio.

\section{CONTROL REGIONS}

As illustrated in Fig. 3 the operating area of a wind turbine can be subdivided into three regions. In region I, below the cut-in wind speed $v_{c u t-i n}$, the turbine does not generate power. When wind speeds become higher than the cut-in speed $v_{c u t-i n}$ (region II), the turbine starts operating in the normal wind zone. Above the rated wind speed $v_{r}$ (region III), the power and rotor speed are kept constant at their rated values. If the wind speed rises above the cut-out speed $v_{\text {cut-out }}$, the braking system brings the rotor to a standstill. The power in the whole operating area of the wind turbine is given by (1), where $\rho$ is the air density, $C p(\lambda, \theta)$ is the power coefficient, $r$ is the rotor radius and $v$ is the wind speed. The power coefficient is function of the tip speed ratio and pitch angle. $C_{p}^{\max }$ is the maximum power coefficient obtained at the optimum pitch angle $\theta_{o p t}$ and tip speed ratio $\lambda_{o p t}$.

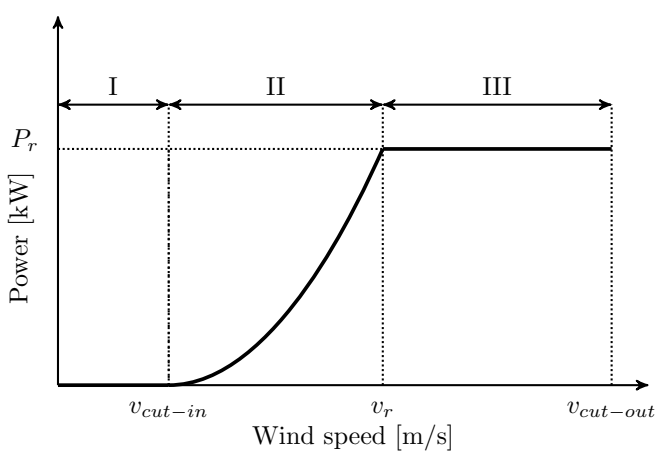

Fig. 3. Wind turbine power curve and operational regions.

$$
P_{m}(v)= \begin{cases}0 & \text { for } v<v_{\text {cut }- \text { in }} \\ \frac{1}{2} \rho \pi r^{2} v^{3} C_{p}^{\text {max }} & \text { for } v_{\text {cut-in }} \leq v \leq v_{r} \\ \frac{1}{2} \rho \pi r^{2} v_{n}^{3} C_{p}(\lambda, \theta) & \text { for } v_{r}<v \leq v_{\text {cut }- \text { out }} \\ 0 & \text { for } v>v_{\text {cut }- \text { out }}\end{cases}
$$

\section{A. Low wind speeds (region II)}

Between the cut-in wind speed and rated wind speed, the control objective is to maximise energy capture over the whole range of wind speeds. As illustrated in Fig. 2, the power coefficient of the wind turbine has a maximum value at the optimum pitch angle and tip speed ratio $\left(C_{p_{\max }}\left(\theta_{\text {opt }}, \lambda_{\text {opt }}\right)\right)$. Therefore, a Maximum Power Point Tracking (MPPT) algorithm is used to keep the aerodynamic efficiency at its maximum value. For this, the pitch angle is fixed to its optimum value, and the constant tip speed ratio is obtained by varying the rotor speed, which must be controlled proportional to the wind speed.

A cascaded control system, consisting of a slow outer and fast inner control loop, is used to track the MPP. The outer power controller provides the reference current to the inner current controller, which controls the generator current by means of the rectifier control. The reference signal is generated using pre-defined power-speed curves obtained through simulations. Because of the wind measurement limits, i.e., anemometer accuracy and possible calibration error, the MPPT algorithm is designed so that a wind speed measurement is not required in the control loop. A cascade controller is designed to control the generator speed to obtain the powerspeed curves at each wind speed. Fig. 4 shows the mechanical power $P_{m}$ and generator power output $P_{e}$ for wind speeds in region II at the optimal pitch angle of $7^{\circ}$. As shown in Fig. 4, if the wind turbine operates at its maximum mechanical power, the power coefficient is not maximised because of the difference between the electrical and mechanical MPP, 
which is caused by the electrical losses in the generator and converter [13]. Therefore, the algorithm must track the electrical MPP as a reference signal to reach a maximum efficiency. The relationship between electrical and mechanical power is defined in (2), where $\eta_{g}$ and $\eta_{c}$ are the generator and converter efficiencies respectively.

$$
P e=\eta_{g} \eta_{c} P_{m}
$$

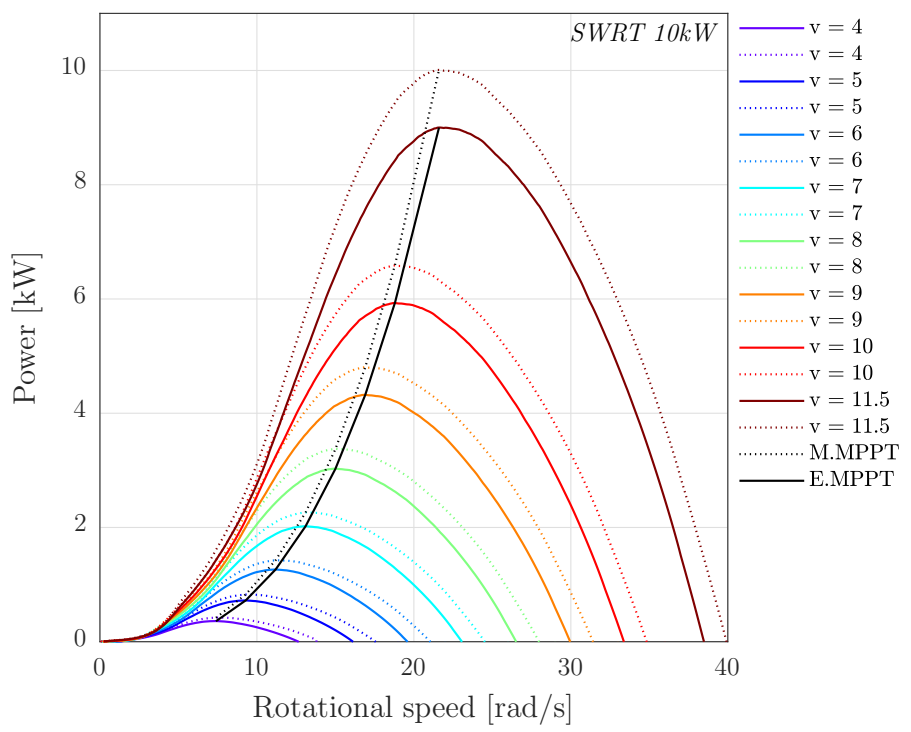

Fig. 4. Electrical versus mechanical power-speed curves.

\section{B. High wind speeds (region III)}

a) Active pitch control: In the pitch controlled wind turbine, the generator speed is limited to its nominal value by pitching the blades to feather. The controller increases the pitch angle, thus reducing the angle of attack, to limit the aerodynamic loads on the blade. When the wind falls below the nominal wind speed, the blades are turned back into their optimum pitch position $\theta_{\text {opt }}$ at which the maximum lift force is produced. The control block diagram of the pitch system is illustrated in Fig. 5. A PI controller regulates the rotor speed and generates the reference pitch signal. This signal is sent to the pitching actuator, which is modelled by a time constant $T_{a}$ of $0.1 \mathrm{~s}$ and a rate limiter of $10 \%$ to ensure that the actuator limits are not exceeded.

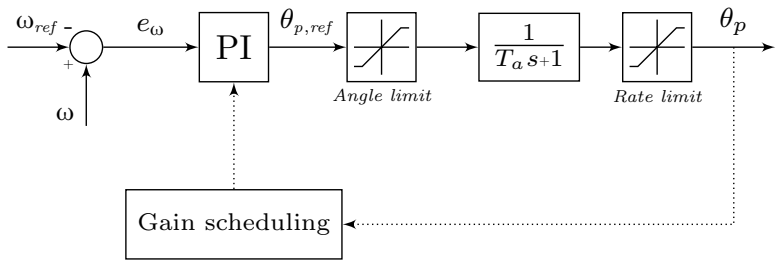

Fig. 5. Pitch control block diagram.
As illustrated in Fig. 6, the sensitivity of aerodynamic power to the pitch angle variation $\left(\frac{\partial P}{\partial \theta}\right)$ is not constant in function of wind speed, as the turbine rotor is a non-linear system. Therefore, a gain-scheduled PI controller is used to compensate for this. The pitch sensitivity of the $10 \mathrm{~kW}$ wind turbine is calculated in the high wind zone by performing a linearisation analysis in FAST. In the linearisation analysis, the pitch angle is altered at the corresponding wind speed, and the variation in aerodynamic power is simulated. It is assumed that the pitch sensitivity increases linearly with the pitch angle, thus:

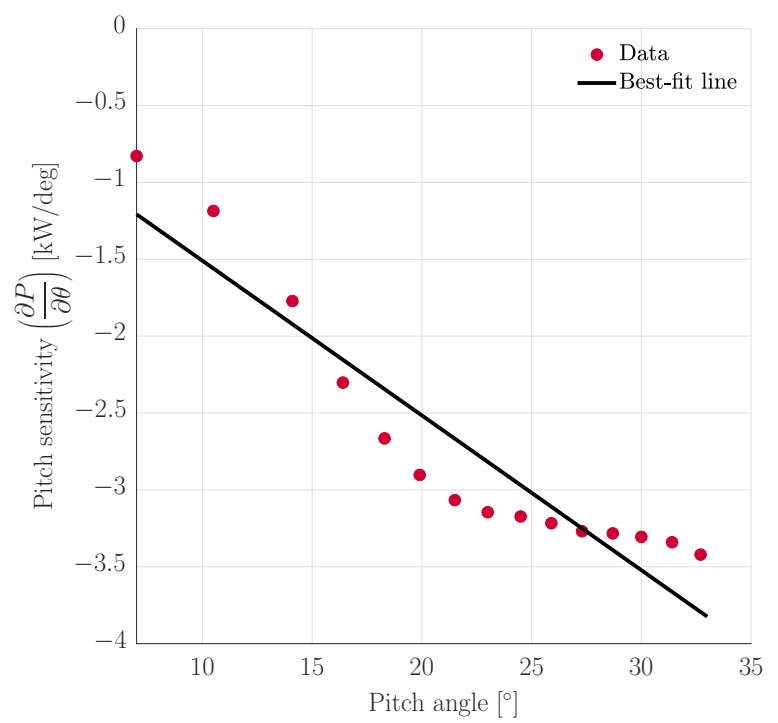

Fig. 6. Pitch sensitivity of the $10 \mathrm{~kW}$ wind turbine in region III.

$$
\frac{\partial P}{\partial \theta}=\left(\frac{\frac{\partial P}{\partial \theta}\left(\theta_{r}\right)}{\theta_{k}}\right) \theta+\left(\frac{\partial P}{\partial \theta}\left(\theta_{r}\right)\right)
$$

where $\frac{\partial P}{\partial \theta}\left(\theta_{r}\right)$ is the pitch sensitivity at the rated wind speed. $\theta_{k}$ is the blade pitch at which blade sensitivity has increased by a factor of 2 from its value at the rated operating point [14].

$$
\frac{\partial P}{\partial \theta}\left(\theta=\theta_{k}\right)=2 \frac{\partial P}{\partial \theta}\left(\theta=\theta_{r}\right)
$$

Based on the linear relation between pitch sensitivity and pitch angle (3), the gain scheduling is implemented by defining a dimensionless gain-correction factor $G_{k}$ as a function of pitch angle [14] [15].

$$
G_{k}=\frac{1}{1+\frac{\theta}{\theta_{k}}}
$$

which can be written in a general form for non-zero optimal pitch wind turbines as: 


$$
G_{k}=\frac{1}{1+\frac{\theta-\theta_{r}}{\theta_{k}}}
$$

According to (6), the controller gain becomes smaller with increasing pitch angle when the turbine operates at high wind speeds.

b) Active speed stall control: In the active speed stall strategy, the wind turbine operates without a pitch mechanism. Therefore, the blades are fixed to the hub with the pitch angle at which the maximum aerodynamic efficiency is reached $\theta_{\text {opt }}$.

In this control concept, the aerodynamic performance of the wind turbine is controlled by reducing the tip speed ratio. The rotor speed can be actively controlled by regulating the torque of the generator in both region II and III. Above the rated wind speed, the generator torque is increased to reduce the rotor speed. Therefore, the wind turbine operates at a lower tip speed ratio which results in a lower aerodynamic efficiency. In other words, the rotor speed is reduced in high winds to force the blades into the stall condition.

Different control objectives can be achieved, e.g., limiting the speed, power or generator torque. According to [16], power limiting produces an energy yield comparable to the pitch control concept. Therefore, the active speed stall control system is developed to limit generator power. Fig. 7 shows the control block diagram of the active speed stall control. A cascade control system is used to control the electrical power. The outer power controller (slow controller) provides the reference signal to the inner speed controller (fast controller), which regulates the generator current.

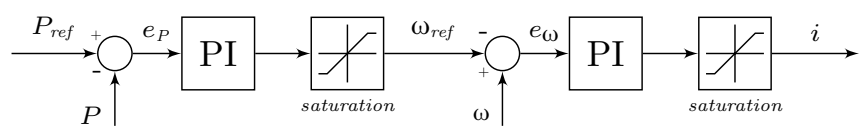

Fig. 7. Active speed stall control block diagram.

The disadvantage of speed stall control is that the generator torque must increase to reduce the rotor speed above the rated wind speed. Therefore, the generator must provide a higher torque than the torque produced at rated power, which means the generator must be overrated [16] [17]. For the $10 \mathrm{~kW}$ wind turbine running up to a cut-out wind speed of $25 \mathrm{~m} / \mathrm{s}$, a generator and a converter with a rated power of $14.8 \mathrm{~kW}$ are needed to limit the power in high winds.

\section{CONTROL PERFORMANCE ANALYSis}

\section{A. Step response}

Fig. 8 shows the performance of the wind turbine operating with both control concepts with a uniform wind speed stepped up from $4 \mathrm{~m} / \mathrm{s}$ to $25 \mathrm{~m} / \mathrm{s}$. In the low wind speeds, the pitch angle is kept at the optimum value $\left(\theta_{\text {opt }}=7^{\circ}\right)$, and the generator torque is actively regulated so that the optimum tip speed ratio $\left(\lambda_{o p t}=5.5\right)$ is obtained. As shown in Fig. 8, the power coefficient is kept at its maximum value $\left(C_{p}^{\max }=0.42\right)$ in region II, which indicates that the MPPT is performing effectively.

When the pitch regulated wind turbine starts operating in the high wind zone, the rotational speed is kept constant until the rated electrical power of $9 \mathrm{~kW}$ is reached. For higher wind speeds, the pitch controller keeps the generator speed at the rated value, and electrical power is controlled by the generator torque. The identical performance of the generator speed and electrical power is obtained over a wide range of wind speeds through scheduling the PI controller gains. The overshoot and oscillations in the generator speed are limited, and the electrical power is kept at the nominal value. The pitch angle varies from $7^{\circ}$ to $34^{\circ}$ in overall to limit the generator speed up to the wind speed of $25 \mathrm{~m} / \mathrm{s}$.

When using active speed stall control, the generator speed is kept constant in the high wind zone until the rated electrical power of $9 \mathrm{~kW}$ is reached. To limit the power to $9 \mathrm{~kW}$, the rotor speed must be reduced to decrease the aerodynamic efficiency of the wind turbine. Therefore, the rotor speed is reduced by increasing the torque of the generator. As illustrated in Fig. 8, the constant power regulation is feasible and stable for both control systems, though the wind turbine operating with active speed stall control presents a higher overshoot and settling time compared to the pitch regulated wind turbine.

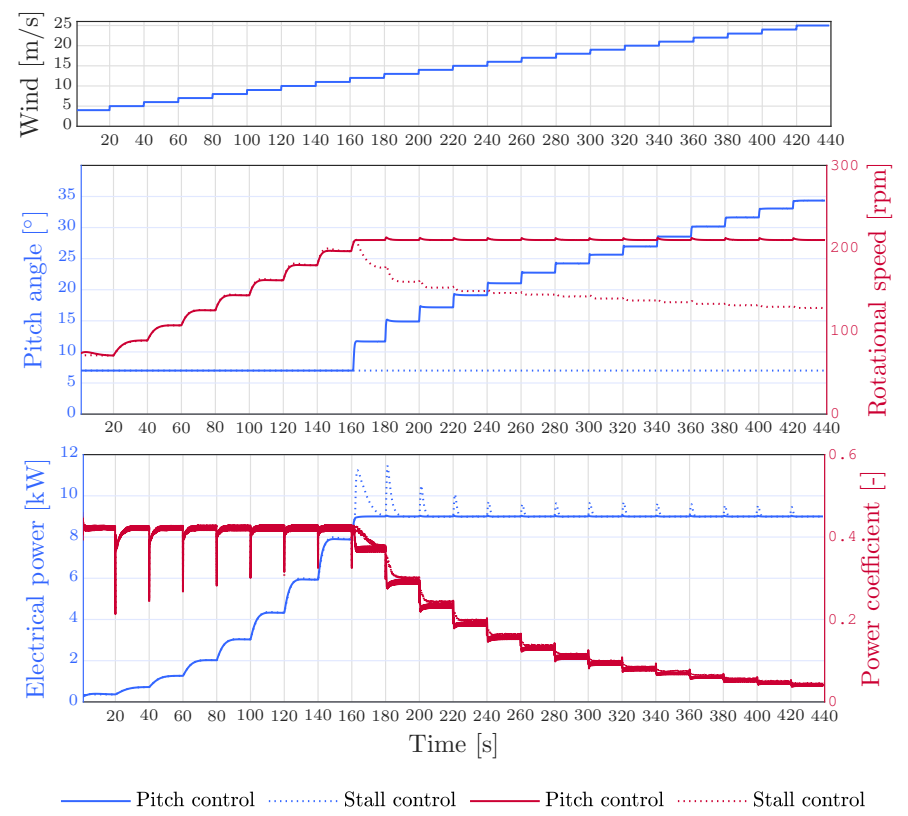

Fig. 8. Performance of the wind turbine in the whole wind range.

\section{B. Turbulent wind speed simulation}

Fig. 9 and Fig. 10 reflect the same parameters as Fig. 8, but for the wind turbine operating in realistic turbulent wind conditions. The wind profile is generated using the TurbSim simulator [18]. The turbulent model of NREL National Wind Technology Center Model (NWTCUP) is selected, and two wind profiles are simulated with mean speeds of respectively $7 \mathrm{~m} / \mathrm{s}$ and $20 \mathrm{~m} / \mathrm{s}$ to assess the control performance in both 
the operating areas II and III. The vertical mean flow (uptilt) angle of the wind is equal to $8^{\circ}$, and horizontal mean flow (skew) angle is $15^{\circ}$.

The performance of the wind turbine in the turbulent wind below the rated wind speed, i.e., with a mean value of $7 \mathrm{~m} / \mathrm{s}$, is shown in Fig. 9. The MPPT algorithm actively adjusts the rotor speed proportional to the wind speed to reach the optimum tip speed ratio. At the same time, the pitch angle is kept constant to its optimum value. Therefore, the maximum power coefficient is obtained over a wide range of wind speeds in region II, which indicates that the MPPT algorithm properly maximises the energy capture for low wind speeds.

Fig. 10 shows the performance of the wind turbine in the turbulent wind between rated wind speed and cut-out wind speed, i.e., with a mean value of $20 \mathrm{~m} / \mathrm{s}$. The pitch controlled wind turbine actively pitches the blade to keep the generator speed at its nominal value, while the wind turbine with active speed stall control adjusts the rotor speed to limit the power. The power output of the pitch regulated turbine is compared to the same turbine with active speed stall control. As illustrated in Fig. 10, both control strategies effectively control the electrical power around the nominal value. However, the pith regulated wind turbine presents smaller fluctuations compared to the wind turbine with active speed stall control.

The constant power concept is perfectly feasible and stable with pitch control, while the active speed stall control is not fast enough to completely handle the extreme changes in the turbulent wind. Since the rotor deceleration is much slower than pitching the blades, the speed stall controller results in higher power oscillations compared to the pitch controller.

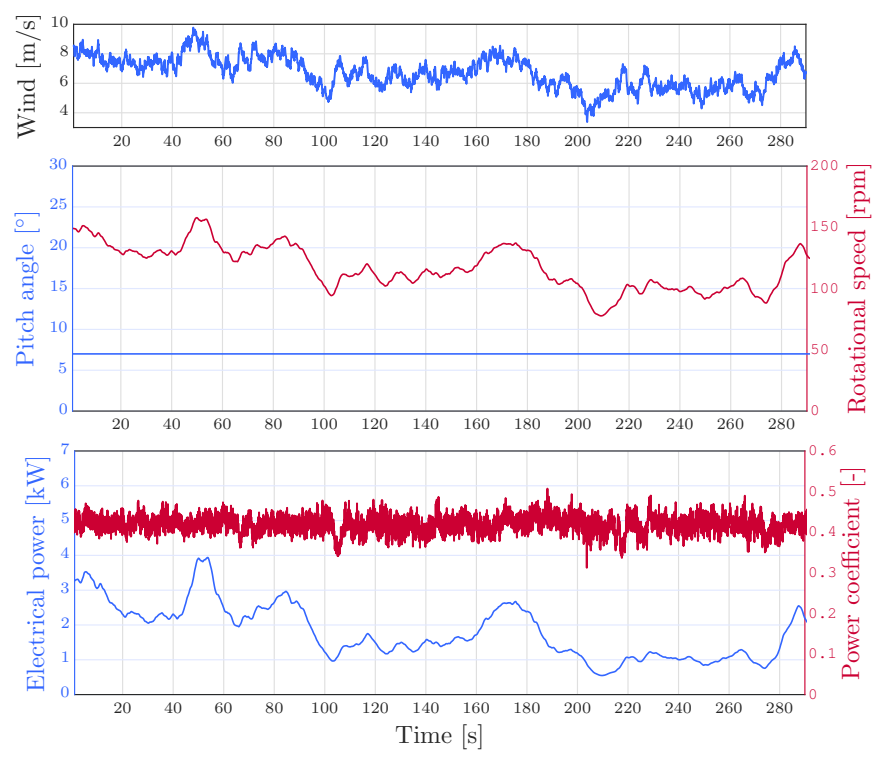

Fig. 9. Performance of the wind turbine in turbulent wind below the rated wind speed (region II).

\section{LOAD ANALYSIS}

The load analysis is performed to investigate the impact of the two different control concepts on the structural loading of
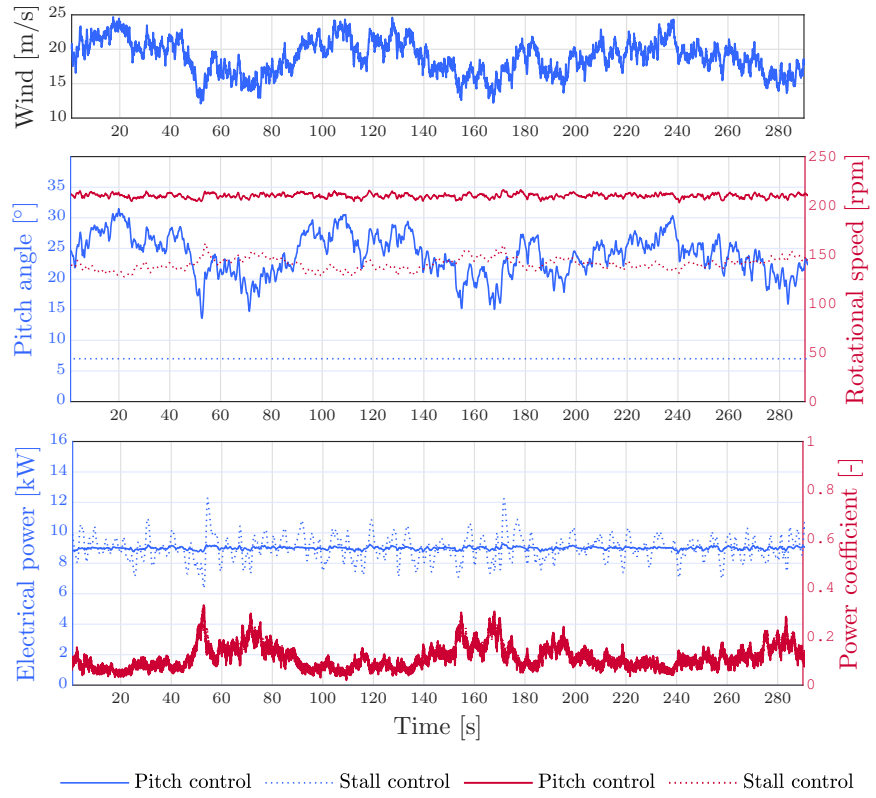

Fig. 10. Performance of the wind turbine in turbulent wind above the rated wind speed (region III).

the rotor blades and the tower of the wind turbine in uniform and turbulent wind. The blade root bending moments, i.e., flapwise and edgewise moments, and tower base bending moments, i.e., fore-aft and side-to-side moments, are monitored. The flapwise moment and the fore-aft moment are caused by the thrust force, which tends to deflect the blades and the tower out of the rotor plane in the downwind direction. The edgewise and side-to-side moments originate from tangential forces, which tend to deflect the blades and the tower in the rotor plane.

The mean load and magnitude of the cyclic components of the fatigue loads are compared in the whole range of wind speeds in Fig. 11. At high wind speeds, the mean load on the structure of the pitch regulated wind turbine is lower than the same wind turbine operating with the active speed stall concept. Therefore, the pitch control has a reduction effect on the static loads, especially in winds above the cut-out wind speed, when the turbine is shut down, and the rotor is in a standstill. As illustrated in Fig. 11, the rotor blades experience higher fatigue loads with active speed stall control. However, the fore-aft and side-to-side moments of the tower base are decreased compared to the pitch control.

Fig. 12 compares the fatigue loads of the blade root and tower base in the turbulent wind with the mean speed of $20 \mathrm{~m} / \mathrm{s}$. Comparing the blade and tower bending loads of the wind turbine running as a pitch regulated wind turbine with the same turbine operating with speed stall control, one can see that the speed stall control lowers the fore-aft and sideto-side fatigue loads on the tower base, while it increases the fatigue load on the rotor blades. 

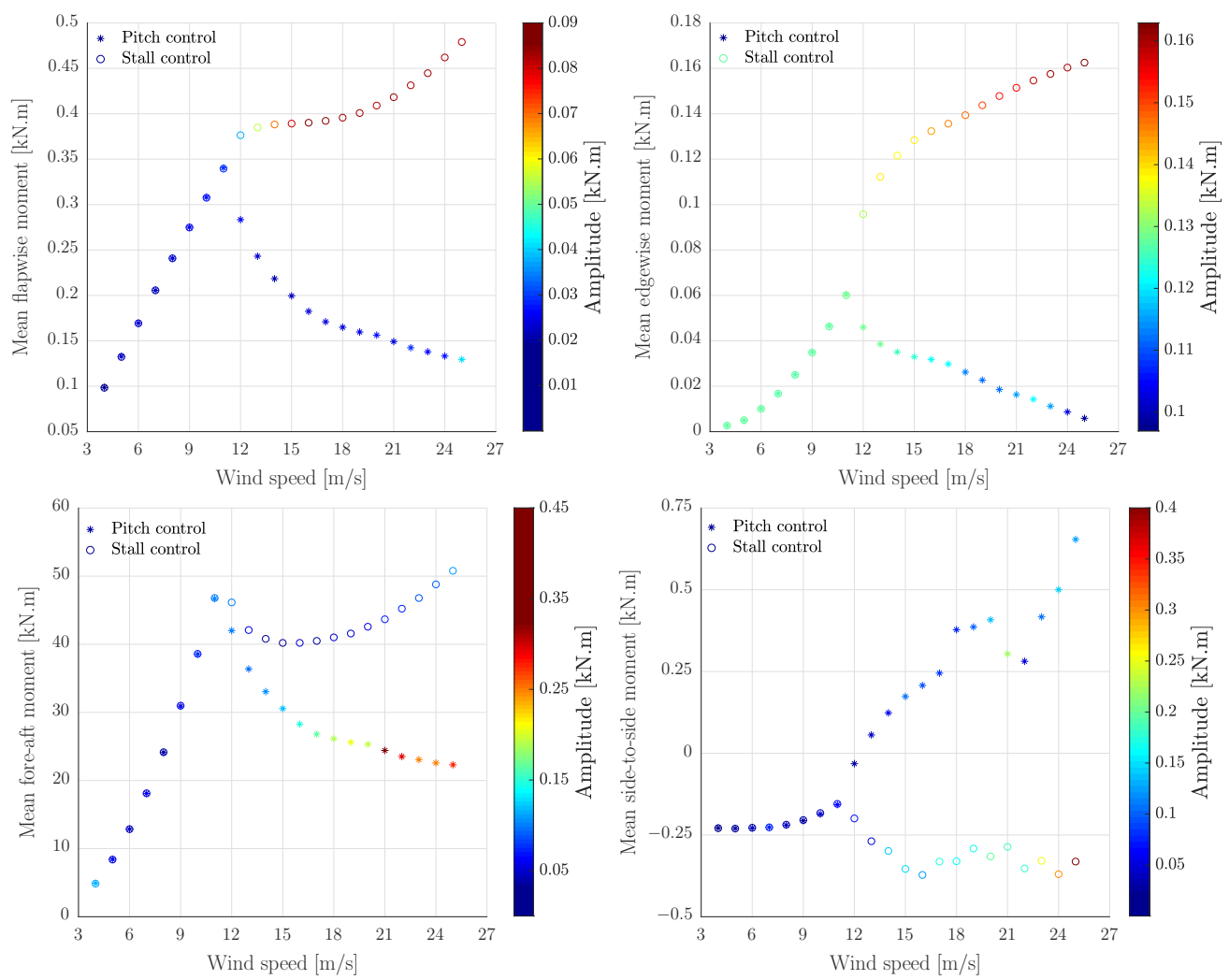

Fig. 11. Comparison of the fatigue load on the structure of wind turbine operating with active speed stall and pitch control in the whole wind speed range.
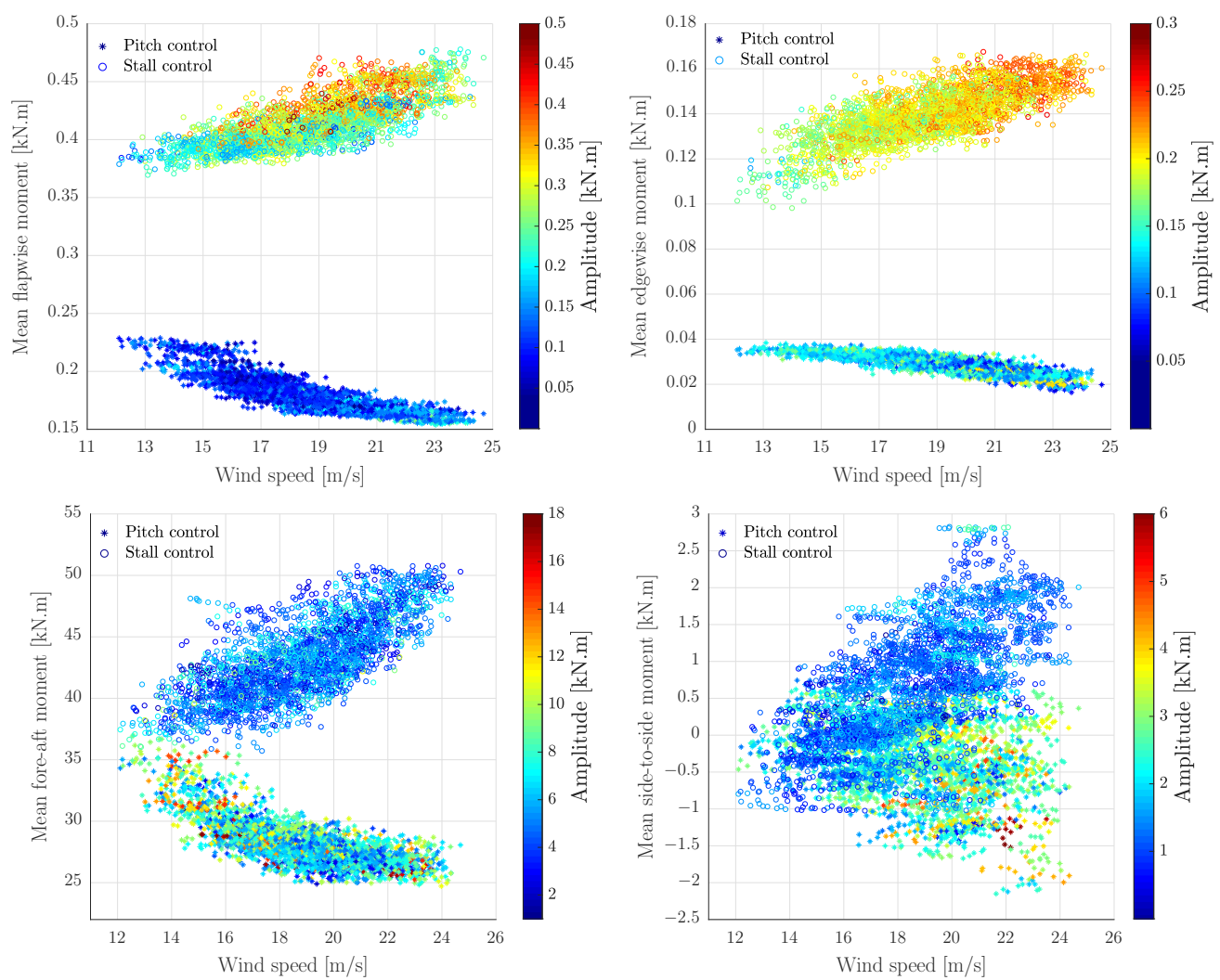

Fig. 12. Comparison of the fatigue load on the structure of wind turbine operating with active speed stall and pitch control concepts in the turbulent wind. 


\section{CONCLUSION}

This paper has investigated the performance of $10 \mathrm{~kW}$ direct-drive PMSG system operating with two control strategies, i.e., pitch control and active speed stall control. In the first control concept, the generator speed is controlled by turning the blades to feather by means of a pitching system. With the active speed stall control, the same wind turbine is operated without a pitch mechanism, and the electrical power is limited by regulating the generator torque. An MPPT algorithm is used to reach the maximum aerodynamic efficiency of the wind turbine in the low wind region, including the generator and converter losses. The performance of the control system is assessed in the whole operating region of the wind turbine for both uniform and turbulent wind conditions. Moreover, the blade root moments and the tower base moments are monitored, and the load analysis is carried out to compare the impact of both control strategies on the structural loads of the wind turbine.

The simulation results have verified that, in the low wind region, the maximum power coefficient is achieved by the proposed MPPT algorithm. At high wind speeds, both active speed stall control and pitch control concepts could limit the electrical power around the rated value. However, speed stall control presents higher power oscillations in the turbulent wind and extreme wind variations. The pitch mechanism reacts faster to wind changes compared to the speed stall control system in which rotor speed is decelerated by regulating the generator torque. According to the load analysis performed for the wind turbine operating in uniform and turbulent wind, it is shown that active speed stall control has a reduction effect on the tower fore-aft and side-to-side fatigue loads, while it increases the blade flapwise and edgewise moments.

\section{ACKNOWLEDGMENT}

This work was performed in the framework of the Catalisti cluster SBO project CO2PERATE, the Energy Transition Fund project BEOWIND of the Belgian federal government, and the FWO research project G.0D93.16N, funded by the Research Foundation Flanders.

\section{REFERENCES}

[1] "Global Annual Installed Capacity of Small and Medium Wind Turbines is expected to exceed 446MW in 2026,"

[5] C. Lumbreras, J. M. Guerrero, P. García, F. Briz, and D. D. Reigosa, "Control of a small wind turbine in the high wind speed region," IEEE Transactions on Power Electronics, vol. 31, no. 10, pp. 6980-6991, 2015. https://www.navigantresearch.com/news-and-views/global-annualinstalled-capacity-of-small-and-medium-wind-turbines-is-expected-toexceed-446-mw-in-2.

[2] Z. Chen, J. M. Guerrero, and F. Blaabjerg, "A review of the state of the art of power electronics for wind turbines," IEEE Transactions on power electronics, vol. 24, no. 8, pp. 1859-1875, 2009.

[3] A. Shafiei, B. M. Dehkordi, A. Kiyoumarsi, and S. Farhangi, "A control approach for a small-scale PMSG-based WECS in the whole wind speed range," IEEE Transactions on Power Electronics, vol. 32, no. 12, pp. 9117-9130, 2017.

[4] J. C. Hui, A. Bakhshai, and P. K. Jain, "An energy management scheme with power limit capability and an adaptive maximum power point tracking for small standalone PMSG wind energy systems," IEEE Transactions on Power Electronics, vol. 31, no. 7, pp. 4861-4875, 2015.

[6] C. Jiawei, W. Changyun, and S. Yongduan, "Power control strategy for variable-speed fixed-pitch wind turbines," in 2014 13th International Conference on Control Automation Robotics \& Vision (ICARCV). IEEE, 2014, pp. 559-564.

[7] Z. M. Dalala, Z. U. Zahid, and J.-S. J. Lai, "New overall control strategy for small-scale WECS in MPPT and stall regions with mode transfer control," IEEE transactions on energy conversion, vol. 28, no. 4, pp. 1082-1092, 2013.

[8] B. Neammanee, S. Sirisumrannukul, S. Chatratana, and S. Muyeen, "Control strategies for variable-speed fixed-pitch wind turbines," in Wind power. InTech, 2010, pp. 209-232.

[9] A. Ahmed, L. Ran, and J. R. Bumby, "New constant electrical power soft-stalling control for small-scale VAWTs," IEEE transactions on energy conversion, vol. 25 , no. 4 , pp. 1152-1161, 2010.

[10] B. Neammanee, S. Sirisumranukul, and S. Chatratana, "Control performance analysis of feedforward and maximum peak power tracking for small-and medium-sized fixed pitch wind turbines," in 2006 9th International Conference on Control, Automation, Robotics and Vision. IEEE, 2006, pp. 1-7.

[11] A. Miller, E. Muljadi, and D. S. Zinger, "A variable speed wind turbine power control," IEEE Transactions on Energy Conversion, vol. 12, no. 2, pp. 181-186, 1997.

[12] B. Jonkman and J. Jonkman, "Fast v8. 16.00 a-bjj," National Renewable Energy Laboratory, 2016

[13] J. D. M. De Kooning, T. L. Vandoorn, J. Van de Vyver, B. Meersman and L. Vandevelde, "Displacement of the maximum power point caused by losses in wind turbine systems," Renewable Energy, vol. 85, pp. 273280, January 2016.

[14] J. Jonkman, S. Butterfield, W. Musial, and G. Scott, "Definition of a 5MW reference wind turbine for offshore system development," National Renewable Energy Lab.(NREL), Golden, CO (United States), Tech. Rep., 2009.

[15] M. H. Hansen, A. D. Hansen, T. J. Larsen, S. Øye, P. Sørensen, and P. Fuglsang, "Control design for a pitch-regulated, variable speed wind turbine," 2005.

[16] D. Bang, H. Polinder, J. Ferreira, and R. Van Rooij, "New active speed stall control compared to pitch control for a direct-drive wind turbine," Europe, vol. 5, no. 11, 2007.

[17] N. Milivojevic, N. Schofield, I. Stamenkovic, and Y. Gurkaynak, "Field weakening control of PM generator used for small wind turbine application," 2011.

[18] B. J. Jonkman and M. L. Buhl Jr, "Turbsim user's guide," National Renewable Energy Lab.(NREL), Golden, CO (United States), Tech. Rep., 2006. 